\title{
Aggressive Posterior Retinopathy of Prematurity Is Associated with Multiple Infectious Episodes and Thrombocytopenia
}

\author{
Pia Lundgren ${ }^{a}$ Linnea Lundberg ${ }^{a}$ Gunnel Hellgren ${ }^{a} \quad$ Gerd Holmström $^{b}$ \\ Anna-Lena Hård ${ }^{a}$ Lois E. Smith ${ }^{e}$ Agneta Wallin $^{c}$ Boubou Hallberg ${ }^{d}$ \\ Ann Hellström ${ }^{a}$ \\ a Section for Ophthalmology, Department of Clinical Neuroscience, Institute of Neuroscience and Physiology, \\ Sahlgrenska Academy, University of Gothenburg, Gothenburg, ${ }^{b}$ Department of Neuroscience/Ophthalmology, \\ University Hospital, Uppsala, and 'St. Eriks Eye Hospital and d Department of Neonatology, Karolinska Institutet

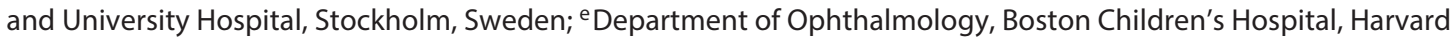 \\ Medical School, Boston, Mass., USA
}

\section{Key Words}

Aggressive posterior retinopathy of prematurity · Preterm infant $\cdot$ Thrombocytopenia $\cdot$ Sepsis

\begin{abstract}
Background: The most severe form of rapidly progressing retinopathy of prematurity (ROP) is termed aggressive posterior ROP (APROP). APROP frequently causes severe visual impairment in affected preterm infants despite timely and appropriate laser treatment. Objectives: We investigated the postnatal characteristics associated with APROP development in a national Swedish cohort. Methods: This retrospective, 1:1 matched case-control study included all infants that developed APROP in zone $1(n=9)$ between 2008 and 2012. Control infants, matched for gestational age and birth weight, developed ROP no worse than stage $2(n=9)$. We retrieved data from medical records on infant birth characteristics, postnatal morbidities, and blood analyses from birth to the first ROP treatment. Infectious episodes included sepsis, C-reactive protein $\geq 10 \mathrm{mg} / \mathrm{l}$, and other clinical signs of infection that required antibiotic treatment. A plate-
\end{abstract}

\section{KARGER}

(C) 2016 S. Karger AG, Basel

E-Mail karger@karger.com

www.karger.com/neo let count $<100 \times 10^{9} /$ I was considered to be thrombocytopenia. Results: All APROP cases postnatally developed at least two infectious episodes, one in the first month and one around the time of ROP diagnosis. All APROP cases exhibited thrombocytopenia in the first month, and 6/9 exhibited thrombocytopenia around the time of ROP diagnosis. Compared to the controls, APROP cases more frequently developed necrotizing enterocolitis $(8 / 9$ vs. $1 / 9 ; p<0.01)$ and sepsis $(9 / 9$ vs. $3 / 9 ; p<0.01)$, and they had significantly lower median platelet counts $\left(90 \times 10^{9} /\right.$, range $4-459$, vs. $158 \times$ $10^{9} /$, range $\left.20-500 ; p<0.001\right)$. Conclusion: Multiple infectious episodes and thrombocytopenia, particularly around the time of ROP diagnosis, were associated with APROP development.

(c) 2016 S. Karger AG, Basel

\section{Introduction}

Retinopathy of prematurity (ROP) is a leading cause of childhood blindness in preterm infants. Immaturity and excessive oxygen administration were the first major 
Table 1. Birth characteristics and postnatal morbidities in infants who developed APROP (cases) and in control infants

\begin{tabular}{lccc}
\hline Characteristic & Cases $(\mathrm{n}=9)$ & Controls $(\mathrm{n}=9)$ & p value \\
\hline GA, weeks + days & $24+1(23+0,26+4)$ & $24+3(22+5,26+6)$ & n.s. $^{2}$ \\
BW, g & $585(470,700)$ & $590(455,635)$ & n.s. $^{2}$ \\
BWSDS & $-0.61(-4.0,0.1)$ & $-1.34(-4.21,0.48)$ & n.s. ${ }^{2}$ \\
BPD & $8(89)$ & $6(67)$ & n.s. $^{3}$ \\
NEC & $8(89)$ & $1(11)$ & $<0.01^{3}$ \\
IVH & $3(33)$ & $1(11)$ & n.s. $^{3}$ \\
Sepsis & $9(100)$ & $3(33)$ & $<0.01^{3}$ \\
\hline
\end{tabular}

Values are presented as the median (min., max.) or $\mathrm{n}(\%)$. BWSDS $=\mathrm{BW}$ standard deviation score; n.s. = nonsignificant.

${ }^{1}$ Calculated with the Scandinavian gender-specific growth reference produced by Marsál et al. [22].

${ }^{2}$ Mann-Whitney U test.

${ }^{3}$ Fischer's exact test. risk factors identified for ROP [1]. Comorbidities, such as bronchopulmonary dysplasia (BPD), necrotizing enterocolitis (NEC), intraventricular hemorrhage (IVH), and sepsis have been associated with ROP [2-4]. In recent years researchers have found that growth factors, such as insulin-like growth factor 1 and vascular endothelial growth factors (VEGF), have important influences in ROP development [5].

ROP displays two phases of development (phases 1 and 2). Phase 1 is characterized by delayed retinal vessel growth, beginning from birth to weeks after birth. Phase 2 is characterized by neovascularization, beginning weeks to months after birth. ROP is classified by stage according to disease severity (ROP stages $0-5$ ) and by zone according to the location of ROP in the retina (zones I to III) [6].

The most severe form of rapidly progressing ROP is termed aggressive posterior ROP (APROP) [6]. APROP is a rare disease. In Sweden between 2008 and 2012, 5.2\% of infants screened for ROP received treatment and of those treated $4.9 \%$ had APROP in zone 1 [7]. APROP is characterized by its posterior location (most commonly observed in zone 1, close to the optic nerve, or in posterior zone 2), prominent vascular activity (neovascularizations and plus disease; i.e. vessel dilation and tortuosity), and rapid, indistinct progression of neovascularization (ROP disease) [6]. APROP treatment may prevent blindness, but the treatment response and visual outcome have been poor, particularly when APROP occurs in zone 1 [8].

Only a few studies have focused on the pathophysiology of APROP. Case reports and clinical experience suggest that APROP develops in infants with the worst health status; however, to our knowledge, only sepsis is identified as a significant risk factor for APROP [9]. Some stud- ies suggest that low platelet counts/thrombocytopenia could be associated with APROP and that platelet transfusion might be a potential new ROP treatment strategy $[10,11]$.

In Sweden, data on all infants screened for ROP are registered in SWEDROP, a national quality register with a $96 \%$ national coverage [7]. In this retrospective, nationbased, 1:1 matched case-control study, we included infants registered in SWEDROP with APROP in zone 1 over a period of 5 years (2008-2012). We determined the postnatal clinical course in preterm infants that developed APROP in zone 1 and in an age- and birth weight (BW)-matched control group, which developed only mild ROP (no worse than stage 2). We aimed to investigate the postnatal characteristics in APROP development.

\section{Methods}

\section{Ethical Considerations}

The study protocol was approved by the Regional Ethics Review Board in Uppsala, Sweden.

\section{Study Population}

The present study included infants registered in SWEDROP with APROP in zone 1, born between January 1, 2008 and December 31, $2012(n=9)$. We performed a retrospective review of medical records from birth to the first ROP treatment. Data on gestational age (GA) and BW were retrieved, as well as the following data on neonatal morbidities: BPD, NEC (medical or surgical interventions, and the number of treatment episodes), IVH, and sepsis (defined as a positive blood culture). We recorded all available data on laboratory measurements of platelet counts and C-reactive protein (CRP) and platelet transfusions. We retrieved data from SWEDROP on ROP status, development, and treatment interventions.
Lundgren et al. 


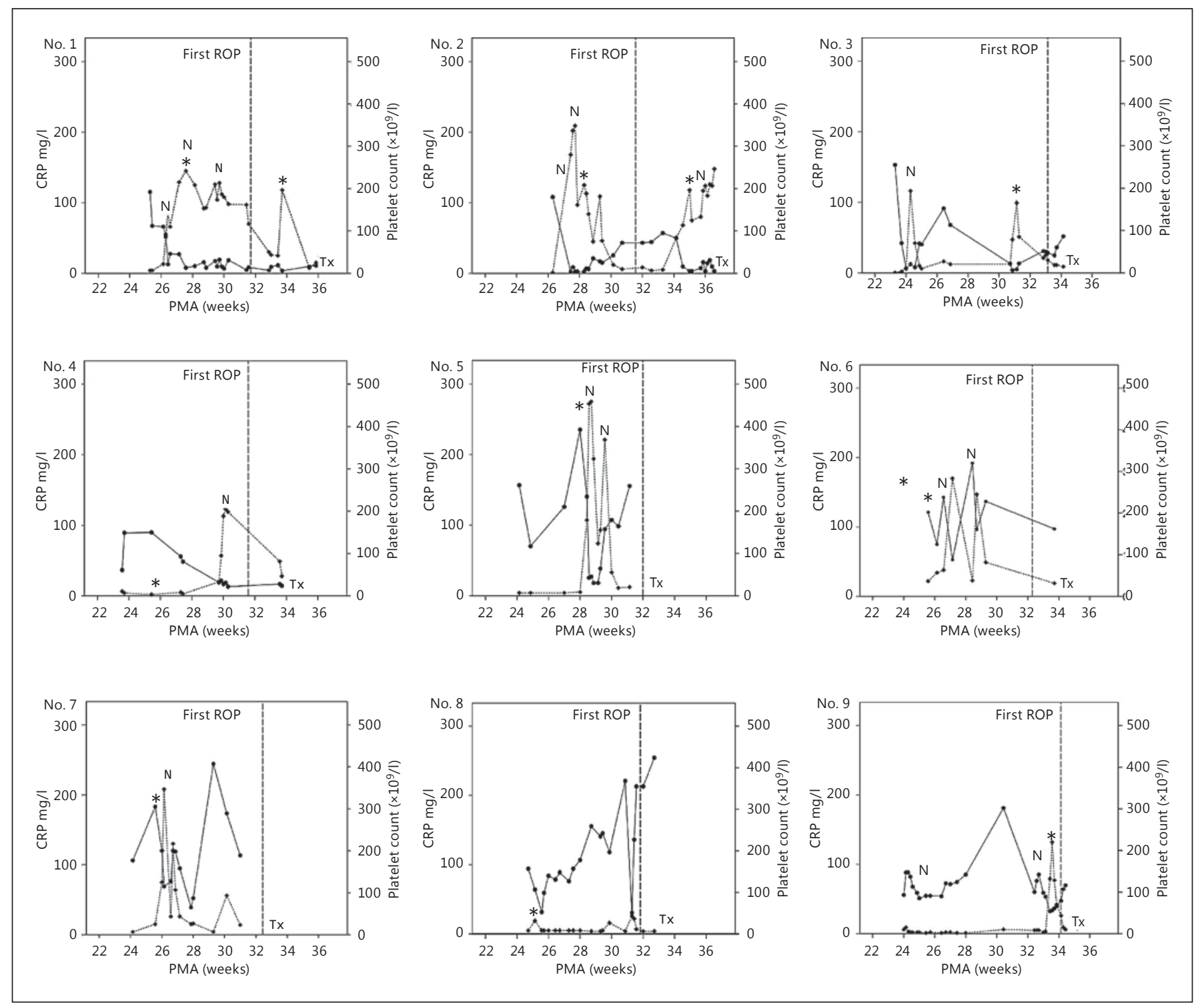

Fig. 1. Serum CRP concentrations (dotted lines) and platelet counts (solid lines) are shown for each individual infant that developed APROP $(n=9)$. Vertical dashed lines indicate the time of the first sign of ROP, and the time of first treatment is also indicated (Tx). N indicates verified NEC; * indicates sepsis.

Each control case was individually matched to an APROP case for BW (within $100 \mathrm{~g}$ ) and GA (within 1 week). Controls were confirmed to have only mild ROP, no worse than ROP stage 2 . For each control case, we retrospectively reviewed the medical records for data equivalent to those retrieved for the APROP cases, up to the postmenstrual age (PMA) when the matched APROP infant received the first ROP treatment.

\section{Neonatal Morbidities and Postnatal Clinical Course}

BPD was defined as the need for continuous supplemental oxygen at 36 weeks PMA [12]. NEC was defined as stage $2 \mathrm{~b}$ or more [13]. IVH included all grades of IVH. Sepsis was defined as a positive blood culture. To describe all events of an infectious character we implemented the term 'infectious episodes', defined as any sepsis, CRP $\geq 10 \mathrm{mg} / \mathrm{l}$, or other clinical signs of infection that required antibiotic treatment.

\section{Platelet Count, CRP and Platelet Transfusions}

All available values for the platelet count and CRP were retrieved from the infants' medical records. When several values were available per day, the lowest platelet count value and the highest CRP value were recorded for that day. National recommendations defined neonatal thrombocytopenia as a platelet count $<100$ $\times 10^{9} / 1$. Data concerning platelet transfusions (volume in milliliters per day) were retrieved. 
Table 2. Comparison of characteristics concerning serum platelet levels and platelet transfusions between infants who developed APROP (cases) and infants who developed mild ROP (controls)

\begin{tabular}{lccc}
\hline Characteristic & Cases $(\mathrm{n}=9)$ & Controls $(\mathrm{n}=9)$ & $\mathrm{p}$ value \\
\hline Total days of platelet count values & $190(10-37)$ & $171(7-37)$ & $\mathrm{n.s.}^{\mathrm{a}}$ \\
Platelet count, $\times 10^{9} / 1$ & $90(4,459)$ & $158(20,500)$ & $<0.001^{\mathrm{b}}$ \\
Days thrombocytopenia values were collected & $56(106 / 190)$ & $27(47 / 171)$ & $<0.001^{\mathrm{a}}$ \\
Total days of platelet transfusions & $16(1-10)$ & $2(1-1)$ & $<0.001^{\mathrm{a}}$ \\
Platelet transfusion, ml/day & $17.5(5,30)$ & $5.5(5,6)$ & $<0.01^{\mathrm{b}}$ \\
\hline
\end{tabular}

Values are presented as $\mathrm{n}$ (range per infant), the median (min., max.) or \% (days/days available). n.s. = Nonsignificant.

${ }^{\mathrm{a}} \chi^{2}$ test. ${ }^{\mathrm{b}}$ Mann-Whitney U test.

\section{ROP Examinations}

All infants were examined according to the established Swedish ROP screening guidelines previously described by Holmström et al. [7]. APROP was diagnosed according the international classification [6], which described it as follows: 'The characteristic features of this type of ROP are its posterior location, prominence of plus disease, and the ill-defined nature of the retinopathy.'

\section{Statistical Analysis}

All statistical analyses, calculations of medians and percentages, and the generation of graphics were carried out with IBM SPSS Statistics 20 for Microsoft Windows (IBM, Armonk, N.Y., USA). The Fisher's exact test, $\chi^{2}$ and Mann-Whitney U tests were used to explore the relationships between variables.

\section{Results}

\section{Birth Characteristics}

The birth characteristics of the 9 APROP cases and their 9 matched controls are shown in table 1 .

\section{Neonatal Morbidities and Postnatal Clinical Course}

The neonatal morbidities and postnatal clinical course of the 9 APROP cases and their 9 matched controls are shown in table 1. NEC and sepsis occurred significantly more frequently among APROP cases than among controls ( $\mathrm{p}<0.01$ and $\mathrm{p}<0.01$, respectively). All APROP cases had at least two postnatal infectious episodes before the first ROP treatment. One episode occurred within the first month of life and the other occurred around the time of the first sign of ROP and/or before the APROP diagnosis. Clinical signs of infection initiated antibiotic treatment without increased CRP or a positive blood culture in 3 cases: 1 (No. 9) at the time of NEC and 2 (No. 4 and 6 ) at the time of clinical signs of respiratory infection. Of the 9 controls, 5 had an infectious episode within the first month of life, and 2 had later infectious episodes.

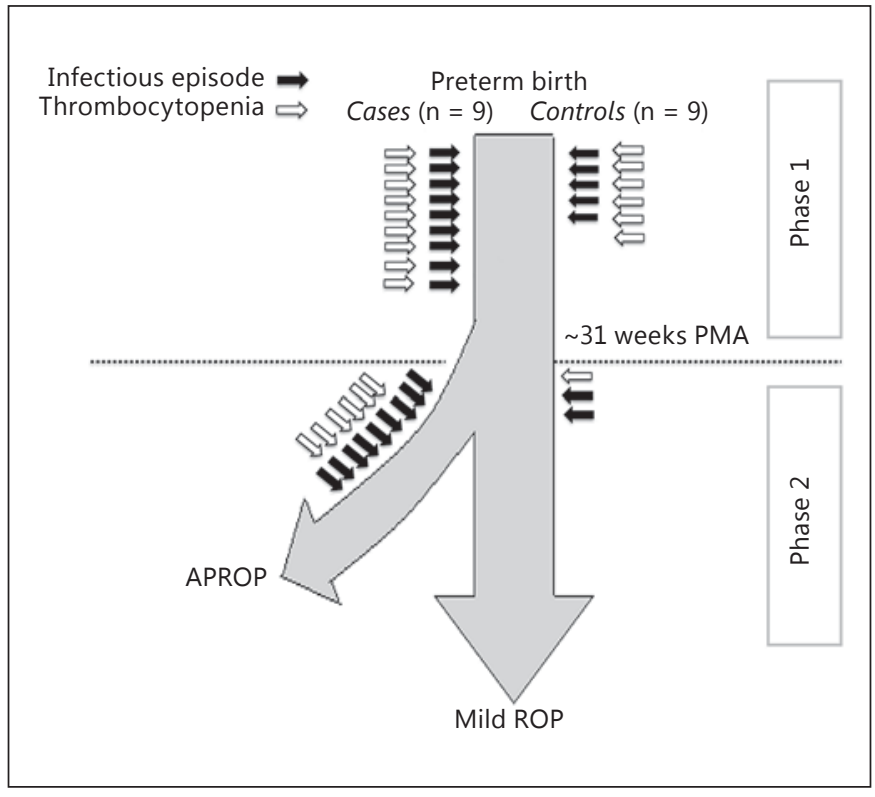

Fig. 2. Graphic representation of the 9 infants that developed APROP (cases) and the 9 age- and BW-matched infants that developed only mild ROP (controls). The horizontal line represents the transition from phase 1 to phase 2 of ROP development. The large gray arrow shows that the clinical signs differed significantly between the two groups during phase 2. The small arrows represent infants with one or more infectious episode events (black arrows) or infants with one or more events of thrombocytopenia (white arrows).

\section{Platelet Count, CRP and Platelet Transfusions}

All 9 APROP cases had an initial period of thrombocytopenia within the first month of life. In addition, 6 APROP cases (No. 1-4, 8, and 9) had another episode of thrombocytopenia around the time of the first signs of ROP and the APROP diagnosis (PMA 31-34 weeks). Four APROP cases (No. 1-4) had persistent 
Table 3. Timing and infant characteristics at the first sign of ROP and ROP treatment interventions in infants that developed APROP

\begin{tabular}{|c|c|c|c|c|c|c|c|c|c|}
\hline 2 & $26+1$ & $\begin{array}{l}27+3,27+4 \\
27+5,34+6 \\
35+5,36+0 \\
36+1,36+3 \\
36+4,36+5\end{array}$ & 2 & $31+4$ & $36+5$ & $2 / 2$ & $2 / 1$ & $1 / 1$ & $-/ 1$ \\
\hline 3 & $23+1$ & $24+0,24+1$ & 2 & $33+1$ & $34+2$ & $1 / 1$ & - & $1 / 1$ & - \\
\hline 4 & $23+2$ & - & 3 & $31+4$ & $34+5$ & $1 / 1$ & - & $1 / 1$ & - \\
\hline 5 & $24+1$ & $29+2$ & APROP & $32+0$ & $32+2$ & $3 / 3$ & $2 / 2$ & $1 / 1$ & - \\
\hline 6 & $23+0$ & - & 1 & $32+2$ & $34+2$ & $3 / 3$ & $1 / 1$ & $2 / 2$ & - \\
\hline 7 & $24+1$ & - & APROP & $32+3$ & $32+5$ & $3 / 3$ & $1 / 1$ & $2 / 2$ & - \\
\hline
\end{tabular}

$\mathrm{L}=$ Left eye; $\mathrm{R}=$ right eye.

thrombocytopenia that lasted for several weeks (fig. 1). Of the 9 controls, 6 developed thrombocytopenia within the first month of life. Only 1 control had an additional period of thrombocytopenia at or after a PMA of 31 weeks. Figure 2 presents a schematic diagram of these results. Days of thrombocytopenia $(\mathrm{p}<0.001)$ and days of platelet transfusion $(\mathrm{p}<0.001)$ occurred significantly more frequently among APROP cases. Median platelet counts $(\mathrm{p}<0.001)$ were significantly lower and median platelet volumes $(\mathrm{ml} /$ day) transfused $(\mathrm{p}<$ 0.01 ; table 2) significantly higher in APROP cases. In table 3 the timing of platelet transfusions in APROP cases are presented. APROP case 2 received numerous platelet transfusions in a total volume of $195 \mathrm{ml}$, but a stable increase in platelet count was not achieved. Of the 9 controls, 2 received a platelet transfusion during their first week of life.

\section{ROP Development and ROP Treatment}

In table 3, the timing and characteristics at the first sign of ROP and ROP treatment interventions in APROP cases are presented. ROP treatment was provided for all APROP cases at a median PMA of 34 weeks and 2 days (range $32+2$ to $36+5$ ). The median number of treatments for APROP was 3 treatment sessions per eye (range 1-5). APROP cases often received a combination of treatment interventions in the same session. Among the 9 control cases, 1 had no ROP, 3 had ROP stage 1 , and 2 had ROP stage 2 .

Aggressive Posterior Retinopathy of Prematurity

\section{Discussion}

APROP is a rare disease, but it generally has a devastating visual outcome, particularly when it appears in zone 1. In this retrospective, nation-based, 1:1 case-control study, we found that multiple infectious episodes and thrombocytopenia in both phases of ROP were associated with APROP development. To the best of our knowledge, this study was the first to suggest this 'multiple-hit pattern' in APROP development. All infants that developed APROP (9/9 cases) had at least one infectious episode and thrombocytopenia within the first month of life in phase 1. Then, within weeks to months after birth, the majority of APROP cases had a second infectious episode and an additional period of thrombocytopenia in phase 2 . In contrast, infants that developed only mild ROP (controls) had infectious episodes and thrombocytopenia less frequently than infants with APROP, particularly in phase 2 .

In agreement with Borroni et al. [9], sepsis was significantly more frequent in APROP cases in our study. However, to describe all events of an infectious character, we implemented the term 'infectious episodes', which included sepsis and any conditions that caused an increase in CRP ( $\geq 10 \mathrm{mg} / \mathrm{l})$ or clinical signs of infection that required antibiotic treatment. All APROP cases displayed at least two postnatal infectious episodes before ROP treatment in both phases of ROP.

Consistent with thrombocytopenia, APROP cases displayed a median platelet count that was significantly 
lower than that of controls, supporting the findings of Vinekar et al. [10] and Jensen et al. [11]. Days of platelet transfusion were significantly more frequent and the median volume platelets transfused was significantly higher in APROP cases compared to the controls. These results could be interpreted as multiple platelet transfusions being a significant risk factor for APROP. However, APROP cases showed a persistent low platelet count despite frequent transfusions and we therefore hypothesize that thrombocytopenia per se is the risk factor for APROP. Vinekar et al. [10] described an infant with APROP and thrombocytopenia that showed spontaneous APROP regression after multiple platelet transfusions and an increase in platelet count, which suggested a potential new ROP treatment strategy if appropriately timed platelet transfusion is effective. Thrombocytopenia is common in neonates, particularly those born extremely preterm with a low BW [14]. A $\geq 30 \%$ drop in platelet counts also without reaching thrombocytopenia as well as thrombocytopenia per se have been correlated and suggested to predict the onset of morbidities and mortality [15]. It is possible that thrombocytopenia alone is associated with APROP, because sepsis can cause low platelet counts [16].

The exact relationship between low platelet counts and ROP remains unclear. Several studies have indicated that platelets are major regulators of angiogenic regulatory proteins, such as VEGF. Platelets store, transport, and deliver VEGF [17]. Increased local and systemic VEGF levels have been detected in the proliferative phase of ROP and primarily at the first detection of ROP [18]. However, it is not clear whether thrombocytopenia disturbs the retinal VEGF balance and how these disturbances might affect each phase of ROP.

In the present study, all APROP cases were affected by one or several comorbidities, including BPD, NEC, and/ or IVH, which are well known to be associated with ROP $[2,3]$. However, only NEC occurred significantly more frequently in APROP cases than in controls. ROP screening is often difficult, especially for infants developing NEC due to their extremely fragile state of health. However, since infants with NEC are at the highest risk of severe $\mathrm{ROP}$ development, we want to emphasize the importance of following screening guidelines for these infants in particular, as suggested previously by van Sorge et al. [19]. The exact relationship between NEC and ROP is unclear. Perplexingly, low oxygen saturation has been found to be a risk factor for NEC [20].

All APROP cases required several ROP treatment sessions, except for 2 infants who were treated with intra- ocular injections of anti-VEGF antibody in an initial treatment. Anti-VEGF treatment has been suggested as the treatment of choice for ROP in zone 1 [21].

The primary strength of our study was that we included a nation-based cohort due to the availability of data in SWEDROP, which tracks infants with complete records during the neonatal period. Another strength was the availability of high-frequency platelet counts in both APROP cases and controls. Although this study was retrospective, the platelet counts were recorded prospectively. Our findings have provided a basis for future, prospective studies, which could investigate the roles of platelets and infectious episodes in the pathogenesis of ROP and APROP.

In conclusion, this study showed that APROP in zone 1 was associated with a 'multi-hit' pattern of pathogenesis. This pattern included infectious episodes and thrombocytopenia in both phases of ROP. Hence, infants with multiple episodes of infection and thrombocytopenia should be given special attention in ROP screening.

\section{Acknowledgements}

We thank the neonatologists who contributed data to the study and whose help was invaluable: Thomas Abrahamsson (Linköping University Hospital), Fredrik Ahlström (Uppsala University Children's Hospital) and Magnus Domellöf (Umeå University Hospital). We thank the board of SWEDROP and all doctors who performed ROP screening in Sweden. This study was supported by the Swedish Medical Research Council (2011-2432), a Swedish government grant (ALFGBG-426531), De Blindas Vänner, the AnnaLisa and Bror Björnsson Foundation, the Carmen and Bertil Regnér Foundation, the Herman Svensson Foundation, the Cronqvists Foundation, Linnéa and Josef Carlssons Foundation, HKH Kronprinsessan Lovisas förening för Barnasjukvård/Stiftelsen Axel Tielmans Minnesfond, NIHEY024864, EY017017, EY022275, P01 HD18655 and Lowy Medical Research Institute, European Commission FP7 project 305485 PREVENT-ROP. The sponsors had no role in the study design, the collection, analysis, and interpretation of data, the writing of the report or the decision to submit the paper for publication.

\section{Disclosure Statement}

The authors declare no conflicts of interest.
Lundgren et al. 


\section{References}

1 Ashton N, Ward B, Serpell G: Effect of oxygen on developing retinal vessels with particular reference to the problem of retrolental fibroplasia. Br J Ophthalmol 1954;38:397-432.

2 Holmstrom G, Broberger U, Thomassen P: Neonatal risk factors for retinopathy of prematurity - a population-based study. Acta Ophthalmol Scand 1998;76:204-207.

3 Arroe M, Peitersen B: Retinopathy of prematurity: review of a seven-year period in a Danish neonatal intensive care unit. Acta Paediatr 1994;83:501-505.

4 Klinger G, Levy I, Sirota L, Boyko V, LernerGeva L, Reichman B: Outcome of early-onset sepsis in a national cohort of very low birth weight infants. Pediatrics 2010;125:e736e740.

5 Hellstrom A, Perruzzi C, Ju M, Engstrom E, Hard AL, Liu JL, Albertsson-Wikland K, Carlsson B, Niklasson A, Sjodell L, LeRoith D, Senger DR, Smith LE: Low IGF-I suppresses VEGF-survival signaling in retinal endothelial cells: direct correlation with clinical retinopathy of prematurity. Proc Natl Acad Sci USA 2001;98:5804-5808.

6 Gole GA, Ells AL, Katz X, Holmstrom G, Fielder AR, Capone A, Flynn JT, Good WG, Holmes JM, McNamara JA, Palmer EA, Quinn GE, Shapiro MJ, Trese MGJ, Wallace DK; Int Comm Classification R: The international classification of retinopathy of prematurity revisited. Arch Ophthalmol 2005;123: 991-999.

7 Holmström G, Hellstrom A, Jakobsson P, Lundgren $P$, Tornqvist $K$, Wallin A: Five years of treatment for retinopathy of prematurity in sweden: results from SWEDROP, a national quality register. Br J Ophthalmol 2016, DOI: 10.1136/bjophthalmol-2015-307263.
8 Vinekar A, Jayadev C, Mangalesh S, Kumar AK, Bauer N, Capone A Jr, Trese M, Shetty B: Comparing the outcome of single versus multiple session laser photoablation of flat neovascularization in zone 1 aggressive posterior retinopathy of prematurity: a prospective randomized study. Retina (Philadelphia) 2015; 35:2130-2136.

9 Borroni C, Carlevaro C, Morzenti S, De Ponti E, Bozzetti V, Console V, Capobianco S, Tagliabue PE: Survey on retinopathy of prematurity (ROP) in italy. Ital J Pediatr 2013;39:43.

10 Vinekar A, Hegde K, Gilbert C, Braganza S, Pradeep M, Shetty R, Shetty KB: Do platelets have a role in the pathogenesis of aggressive posterior retinopathy of prematurity? Retina (Philadelphia) 2010;30:S20-S23.

11 Jensen AK, Ying GS, Huang J, Karp K, Quinn GE, Binenbaum G: Thrombocytopenia and retinopathy of prematurity. J AAPOS 2011; 15:e3-e4.

12 van Marter LJ: Epidemiology of bronchopulmonary dysplasia. Semin Fetal Neonatal Med 2009; 14:358-366.

13 Walsh MC, Kliegman RM: Necrotizing enterocolitis: treatment based on staging criteria. Pediatr Clin North Am 1986;33:179-201.

14 Christensen RD, Yoder BA, Baer VL, Snow GL, Butler A: Early-onset neutropenia in small-for-gestational-age infants. Pediatrics 2015;136:e1259-e1267.

15 Rastogi S, Olmez I, Bhutada A, Rastogi D: Drop in platelet counts in extremely preterm neonates and its association with clinical outcomes. J Pediatr Hematol Oncol 2011;33: 580-584.

16 Guida JD, Kunig AM, Leef KH, McKenzie SE, Paul DA: Platelet count and sepsis in very low birth weight neonates: is there an organismspecific response? Pediatrics 2003;111:14111415 .
17 Italiano JE Jr, Richardson JL, Patel-Hett S, Battinelli E, Zaslavsky A, Short S, Ryeom S, Folkman J, Klement GL: Angiogenesis is regulated by a novel mechanism: pro- and antiangiogenic proteins are organized into separate platelet alpha granules and differentially released. Blood 2008;111:1227-1233.

18 Hellgren G, Lofqvist C, Hard AL, HansenPupp I, Gram M, Ley D, Smith LE, Hellstrom A: Serum concentrations of vascular endothelial growth factor in relation to retinopathy of prematurity. Pediatr Res 2016;79:70-75.

19 van Sorge AJ, Schalij-Delfos NE, Kerkhoff FT, van Rijn LJ, van Hillegersberg JL, van Liempt IL, Peer PG, Simonsz HJ, Termote JU: Reduction in screening for retinopathy of prematurity through risk factor adjusted inclusion criteria. Br J Ophthalmol 2013;97:1143-1147.

20 Stenson BJ, Tarnow-Mordi WO, Darlow BA Simes J, Juszczak E, Askie L, Battin M, Bowler U, Broadbent R, Cairns P, Davis PG, Deshpande S, Donoghoe M, Doyle L, Fleck BW, Ghadge A, Hague W, Halliday HL, Hewson M, King A, Kirby A, Marlow N, Meyer M, Morley C, Simmer K, Tin W, Wardle SP, Brocklehurst P: Oxygen saturation and outcomes in preterm infants. New Engl J Med 2013;368:2094-2104.

21 Spandau U, Tomic Z, Ewald U, Larsson E, Akerblom H, Holmstrom G: Time to consider a new treatment protocol for aggressive posterior retinopathy of prematurity? Acta Ophthalmol 2013;91:170-175.

22 Marsál K, Persson PH, Larsen T, Lilja H, Selbing A, Sultan B: Intrauterine growth curves based on ultrasonically estimated foetal weights. Acta Paediatr 1996;85:843-848. 